\title{
A Performance Testing Platform of ECG Electrodes Based on Millipore Films
}

\author{
Jinzhong Song ${ }^{1,2, *}$, Hao Liu ${ }^{3}$, Ming Wei ${ }^{1,2}$ and Jinsong $\mathrm{Li}^{1}$ \\ ${ }^{1}$ College of Biomedical Engineering \& Instrument Science, Zhejiang University, China \\ ${ }^{2}$ China astronaut research and training center, China \\ ${ }^{3}$ Tianjin Polytechnic University, China \\ ${ }^{*}$ Corresponding author
}

\begin{abstract}
With the continuous development of wearable health monitoring technology, new types of Electrocardiogram (ECG) electrodes become the focus of attention, such as textile electrode, non-contact electrode, and micro-needle electrode. However, there are not yet unified performance testing methods for different types of electrodes. Two evaluating indicators proposed in the national pharmaceutical industry standard YY/T 0196-2005 (disposable ECG electrodes) are DC offset voltage and AC impedance. In this paper, Millipore films were used to simulate the human skin, and a testing platform for quantitatively evaluating the electrode performance was designed, composed of three parts: a simulating skin module, a pressure controlling device and a measuring device, which could simulate the electrochemical performance of the interface between ECG signals and human skin surface. And some quantitatively parameters, obtained with this platform, were impedance spectrum of electrode-skin contact interface, static open circuit voltage and dynamic open circuit voltage, which was very important for ECG electrode performance analysis.
\end{abstract}

Keywords-wearable; electrode; millipore film; offset voltage; AC impedance

\section{INTRODUCTION}

Electrocardiogram (ECG) signals are widely used in patient monitoring, health examination, and family care, which provide an important basis for human heart health examination. ECG signals are usually collected by the wet electrodes, which are used by the conductive paste to ensure a good contact between the skin and electrode, and this way has been widely recognized in the stability and fidelity for ECG signal measurement. With the continuous improvement of people's living standard, health monitoring will not only useful in the hospital but also in the family. As wearable health monitoring has become more and more important, the need of low immunity has become one of the important targets for the development of ECG signal acquisition technology. ECG acquisition by the traditional wet paste electrode $(\mathrm{Ag} / \mathrm{AgCl})$ presents a lot of problems [1], such as inconvenience, allergies.

In recent years, dry electrodes have entered the sight of people, with whom the extraction of biological signals from human body is without skin pretreatment and the conductive paste. Several types of dry electrodes are produced, such as textile electrode, non-contact electrode, and micro needle electrode [2]. And their performance testing has become a key technology, and the national pharmaceutical industry standard YY/T 0196-2005 (disposable ECG electrodes) is usually used by many scholars. However, this standard is no longer suitable for dry electrode performance evaluation, and an effective method for ECG electrode performance testing was presented in this paper, which provided the basis for performance evaluation of a variety of ECG electrode on the market (wet and dry electrode electrode).

\section{YY/T 0196-2005 DISPOSABLE ECG ELECTRODE}

\section{A. The Range}

The people's Republic of China standard YY/T pharmaceutical industry 0196-2005 (disposable ECG electrode) says the mark, the safety, and the performance requirements of the disposable ECG electrode used in ECG diagnosis and monitoring. It says: Any disposable ECG electrode system consisting of sensor elements and electrolytes is included in this standard. While, active electrodes, needle electrodes, reusable electrodes, and electrodes for energy transferring are not included in the standard range.

\section{B. Indicator Definition}

- DC offset voltage

The voltage which is formed between the adhesive and the adhesive electrode pair due to the difference of half-cell potential.

\section{- Impedance}

In response to sinusoidal current, the charge storage capacity (capacitance) of preventing current flowing through the electrode interface (resistance) and electrode interface.

\section{Indicator Requirements}

\section{- AC Impedance}

The impedance average should not exceed $2 \mathrm{k} \Omega$ among 12 pairs of adhesive electrodes at $10 \mathrm{~Hz}$ and no more than $100 \mathrm{uA}$ (peak-peak) current. The impedance for each pair of adhesive electrodes should not exceed $3 \mathrm{k} \Omega$.

Skin resistance varies from hundreds of ohms to hundreds of thousands of ohms.

\section{- DC Offset Voltage}

The DC offset voltage of a pair of adhesive electrodes after the stability period should not exceed $100 \mathrm{mV}$.

As can be seen from the standard, the performance of the disposable electrode is often tested through the pair of 
electrodes. However, this way is no longer applicable for the new type of ECG dry electrodes. The dry electrode is the one that does not require pretreatment of the body skin and using a conductive paste when extracting the body surface bioelectrical signal. This particularity determines the difference of impedance and the offset voltage between the wet and dry electrodes. At the same time, the character between the electrode-skin interfaces for dry electrodes reflects the exchange information in extracting bioelectric signals from the human body surface.

Based on industry standards, the open circuit voltage and AC impedance of the electrode-skin contact interfaces were proposed in this paper. For the variety of ECG electrodes, accurately measuring the skin-contact impedance is very significant.

A performance testing platform for ECG electrodes based on Millipore films was designed in this paper. Millipore films were used to simulate the human skin, and this platform was composed of three parts: a simulated skin module, a pressure controlling device and a measuring device, which could simulate the electrochemical performance of the interface between ECG signals and human skin surface. And some quantitatively parameters, obtained with this platform, were impedance spectrum of electrode-skin contact interface, static open circuit voltage and dynamic open circuit voltage, which was very important for ECG electrode performance analysis.

\section{A PERFormanCE TESTING PlatForm BASED ON MILLIPORE FILM}

A performance testing platform was designed in this paper, which could simulate the properties of human skin and adjust the pressure between the electrode and the skin, the relative movement speed, and the trajectory. What's more, relative motion and contact pressure could be changed through the pressure sensor and the two-dimensional motion platform, some quantitatively parameters, extracted by this platform, were impedance spectrum of electrode-skin contact interface, static open circuit voltage and dynamic open circuit voltage.

\section{A. The Design Principle}

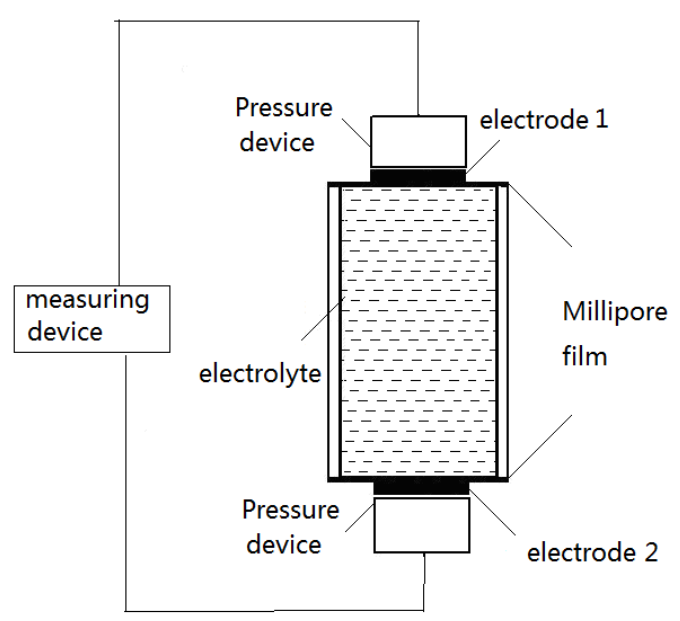

FIGURE I. THE DESIGN PRINCIPLE OF THE PLATFORM
The design principle of the platform is shown in Figure 1, and this platform is composed of a stimulating skin module, a pressure controlling device, and measuring device. The colloidal electrolyte or a liquid electrolyte is used to simulate human skin tissue, then put it into a bucket, and seal the opening in both sides with the Millipore film, which is used as simulated skin membrane. And measuring electrodes are put on the skin surface with the pressure control device, and the relevant parameters are obtained by measuring device, including impedance spectroscopy, static voltage, dynamic open circuit voltage. What's more, some parameters can adjusted with the platform, which including the concentration of electrolyte, the skin membrane pore size, membrane electrode skin pressure, barrel length, relative speed of electrode the skin membrane, the relative movement trajectory between the electrode and skin.

\section{B. The Design of Stimulating Skin Module}

The stimulating skin module is the most critical in the platform, whose principle is shown in Figure 2, the upper and lower microporous membrane with a certain concentration of liquid electrolyte or solid electrolyte are used to simulate two different parts of the human body skin, which is the core component. Confirming the flatness and a certain tension of the film, and preventing liquid leakage are the key part of the design.

The stimulating skin module is mainly composed by the barrel, the simulating film, the ABS disc, and the reference electrode, as shown in Figure 2. In the vertical side of the barrel, there are two threaded holes, which is used to connect the pagoda joints, and then attached to the silicone hose, respectively, correspond to the electrolyte inlet and outlet. Flowing rate of the electrolyte can be displayed in the flow meter in real time. The film is selected from Millipore's hydrophilic film with an effective pore size of $0.1 \mu \mathrm{m}$, a thickness of $0.135 \mathrm{~mm}$ and a diameter of $143 \mathrm{~mm}$. Two pieces of ABS board (thickness:5mm, external diameter:100mm, internal diameter:73mm) are used to simulate the skin clamping, and in the following piece of ABS board there is groove with a diameter of $78 \mathrm{~mm}$ for the placement of seals, and two ABS boards are fixed with nylon screws.
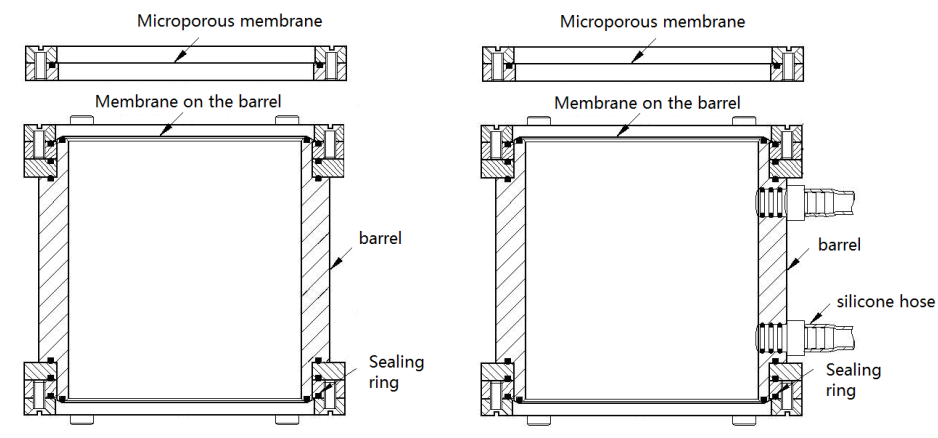

FIGURE II. STIMULATING SKIN MODULE

\section{Pressure Controlling Device}

On the platform there are two pressure controlling devices, which can adjust pressure value between the electrode and the stimulating skin, and the direction is controlled by the rotary 
knob (clockwise for rising, counterclockwise for decreasing). The pressure value between the electrode and the skin can be measured using a pressure sensor (model: CD17-600g, the measurement range: $0-600 \mathrm{cN}$, accuracy: $0.1 \mathrm{cN}$ ).

\section{Measuring Device}

Measuring device in this platform is mainly the electrochemical workstation, which can effectively measure some parameters such as the impedance spectrum, open circuit voltage static and open circuit voltage.

Electrochemical workstation is an instrument composed of constant current meter, constant potential rectifier and electrochemical impedance analyzer, which can not only do the three basic functions of routine test, but also detect the battery voltage, current, capacity and other basic parameters, and it reflects the electrochemical reaction mechanism of AC impedance parameters.

\section{E. The Platform Object}

The 3-D image and physical image of the performance testing platform for ECG electrodes are shown in figure 3.

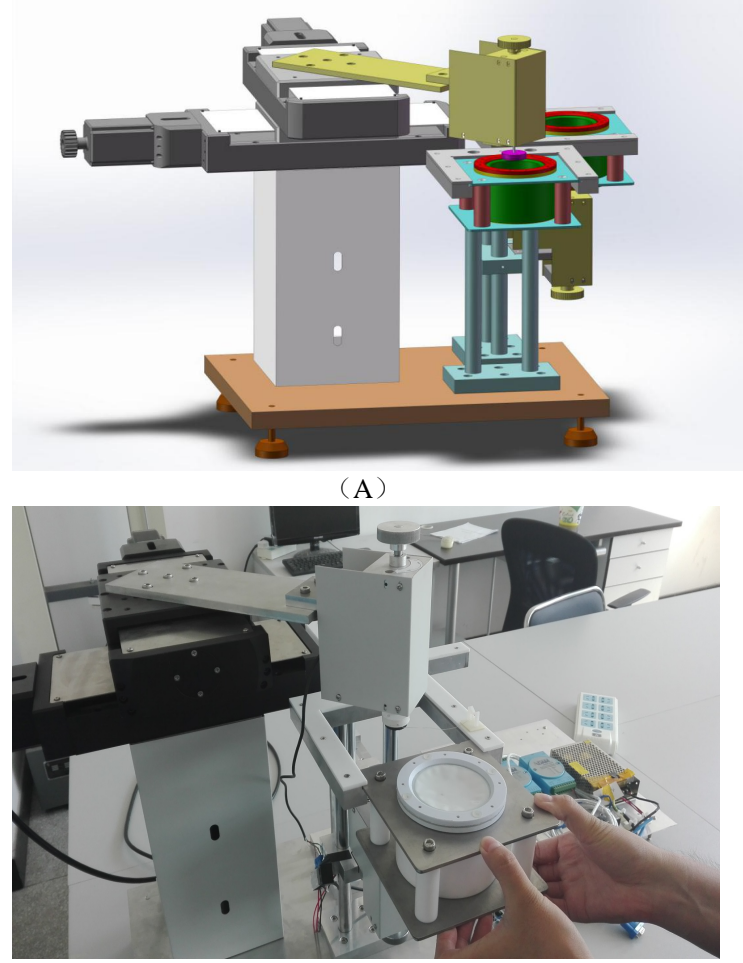

(B)

FIGURE III. THREE-DIMENSIONAL IMAGE AND PHYSICAL IMAGE OF THE PLATFORM IN THIS PAPER

\section{(A) 3-D MODEL, (B) PHYSICAL OBJECT}

\section{DISCUSSION AND CONCLUSION}

With the continuous development of wearable health monitoring technology, new types of Electrocardiogram (ECG) electrodes become the focus of attention, such as textile electrode, non-contact electrode, and micro-needle electrode.
However, there are not yet unified performance testing methods for different types of ECG electrodes.

The platform in this paper is composed of three parts: a simulating skin module, a pressure controlling device and a measuring device. a colloidal electrolyte or a liquid electrolyte is used to simulate human skin tissue, then put it into a bucket, and seal the opening in both sides with the Millipore film, which is used as simulated skin membrane.

The platform in this paper can reflect different electrochemical characteristics of the skin and has a wide adaptability, which can provided a vivid instrument analyzing the correlation among the concentration of electrolytes in the barrel, barrel length, stimulating film size, ECG signal quality and so on. What's more, the platform in this paper can be used to analyzing the correlation between ECG quality and other parameters, such as the pressure of electrode-skin interface, the movement characters of electrodes on the human body surface.

Two evaluating indicators proposed in the national pharmaceutical industry standard YY/T 196-2005 (disposable ECG electrodes) are DC offset voltage and AC impedance, and the two of electrode-skin contact interface are more accurately obtained by this platform, which is a big step forward.

\section{ACKNOWLEDGMENT}

This research was supported in part by National Natural Science Foundation of China (61401417), supported by State Key Laboratory of Space Medicine Fundamentals and Applications (SMFA15A01,SMFA16B06,SMFA13B03), supported by the foundation of China space medicine engineering advanced research (2014SY54A1102, 2015SY54A0501).

\section{REFERENCES}

[1] Song Jinzhong, Chen Hua, Zhang Hui et al. Detection Methods for Skin-electrode Contact Impedance of Textile Electrodes. Progress in Modern Biomedicine. 2015.15(24):4777-4781.

[2] Song Jinzhong, Yan Hong, Xu Zhi et al. Textile Electrode Technologies Applicable to On-orbit Electrocardiogram Acquisition of Astronauts. Space Medicine \& Medical Engineering. 2015,28(6):464-468.

[3] YY/T 0196-2005 disposable ECG electrode. The people's Republic of China standard YY/T pharmaceutical industry. China food and drug administration. 2005.10

[4] Xu Pengjun. Skin-electrode mechanical interaction and motion artifacts of textile electrodes for body surface ECG signal monitoring[D]. Shanghai. Donghua University, Textile Material and Textiles Design,2012:1-19

[5] Bahareh Taji, Shervin Shirmohammadi, Voicu Groza, et al. Impact of Skin-Electrode Interface on Electrocardiogram Measurements Using Conductive Textile Electrodes[J]. IEEE Transactions on Instrumentation and Measurement, 2013, 63(6): 1412-1422

[6] Vojkan M, Benard G. The effect of force and electrode material on electrode-to-skin impedance[C]. Biomedical Circuits and Systems Conference (BioCAS), Hsinchu,Taiwen. 2012, November 28-30: 57-60

[7] Antonio Lanata, Gaetano Valenza. A novel EDA glove based on textile-integrated electrodes for affective computing[J]. Med Biol Eng Comput, 2012, (50):1163-1172

[8] Dilpreet Buxi, Sunyoung Kim, Nick van Helleputte, et al. Correlation Between electrode-tissue Impledance and motion artifact in biopotential recordings[J]. IEEE sensors journal, 2012, 12(12): 3373-3383

[9] Kannaian T, Neelaveni R, Thilagavathi G, et al. Design and Development of embroidered textile electrodes for continuous measurement of electrocardiogram signals[J]. Journal of Industrial 
Textile, 2012, 42(3): 303-318

[10] Li Xie, Geng Yang, Linlin Xu, et al. Characterization of Dry Biopotential electrodes[C]. 35th Annual international conference of the IEEE EMBS. Osaka, Japan. 2013, July 3-7:1478-1481

[11] Gilsoo Cho, Keesam Jeong, Min Joo Paik, et al. Performance Evaluation of Textile-Based Electrodes and Motion Sensors for Smart Clothing[J]. IEEE Sensors Journal, 2011, 11(12): 3183-3192

[12] Catarino A, Carvalho H, Maria J, et al. Continuous Health Monitoring Using E-Textile Integrated biosensors[C]. 2012 international conference and exposition on electrical and power engineerng (EPE 2012). Lasi, Romania. 2012, October 25-27: 605-609

[13] Song H-Y, Lee J-H, Kang D, et al. Textile electrodes of jacquard woven fabrics for biosignal measurement[J]. J Text Inst, 2010, 101(3):768-770

[14] Pola T, Vanhala J. Textile electrode in ECG measurement. In: Proceedings of 3rd International Conference on Intelligent Sensors[C]. Sensor Networks and Information Processing, Melbourne, 2007, 635-639

[15] Baek JY, An JH, Choi JM, et al. Flexible polymeric dry electrodes for the long-term monitoring of ECG[J]. Sensors and Actuators A, 2008, 143(5): 423-429

[16] Westbroek, P, Priniotakis G, Palovuori E, et al. Method for quality control of textile electrodes used in intelligent textiles by means of (EIS) electrochemical empedance spectroscopy[J]. Textile research Journal, 2004, 83(3): 169-174

[17] Kevin C. Tseng, Bor-Shyh Lin, Lun-De Liao, et al. Development of a wearable mobile electrocardiogram monitoring system by using novel dry foam electrodes[J]. IEEE systems Journal, 2013, 22(3): 1-7

[18] Tong InOh, Kyung Hee Univ, Yongin, et al. Nanofiber web textile dry electrodes for long-term biopotential recording[J]. IEEE Transaction on Biomedical circuits and systems, 2013, 7(2): 204-211

[19] D.Swanson and J. Webster. A model for skin-elecrode impedance[C]. Academic Press. New York, USA. 1974, April 2-5: 117-128

[20] Gordon Mark Paul, Fan Cao, Russel Torah, et al. A Smart Textile Based Facial EMG and EOG Computer Interface[J]. Ieee Sensors Journal, 2014, 14( 2):393-400

[21] Jinzhong Song, Hong Yan, Yanjun LI, et al. Research on Electrocardiogram Baseline Wandering Correction Based on Wavelet Transform, QRS Barycenter Fitting, and Regional Method[J]. Australasian Physical \& Engineering Sciences in Medicine, 2010, 33(3): 243-250

[22] Jinzhong Song, Hong Yan, Xinming Yu, et al. Relationship Among QRS Complex Characters in Electrocardiogram and Its Application to Myocardial Ischemia[J]. Journal of Mechanics in Medicine and Biology, 2012,11(5):1103-1111

[23] Kang-Hwi Lee, Jeong-Whan Lee, Woo-Chul Jung, et al. Asymmetric Skin-to-Electrode Impedance Characteristics of Concentric Circular Ring Electrode for Monitoring of Electrical Activity of the Heart[C]. SICE-ICASE International Joint Conference 2006. Busan ,Korea. 2006, Oct 18-21:1135-1136 